\title{
Construction of Special Solutions for Nonintegrable Systems
}

\author{
Sergey Yu VERNOV \\ Skobeltsyn Institute of Nuclear Physics, Moscow State University, \\ Vorob'evy Gory, 119992 Moscow, Russia. \\ E-mail:svernov@theory.sinp.msu.ru
}

Received April 8, 2005; Accepted in Revised Form August 29, 2005

\begin{abstract}
The Painlevé test is very useful to construct not only the Laurent series solutions of systems of nonlinear ordinary differential equations but also the elliptic and trigonometric ones. The standard methods for constructing the elliptic solutions consist of two independent steps: transformation of a nonlinear polynomial differential equation into a nonlinear algebraic system and a search for solutions of the obtained system. It has been demonstrated by the example of the generalized Hénon-Heiles system that the use of the Laurent series solutions of the initial differential equation assists to solve the obtained algebraic system. This procedure has been automatized and generalized on some type of multivalued solutions. To find solutions of the initial differential equation in the form of the Laurent or Puiseux series we use the Painlevé test. This test can also assist to solve the inverse problem: to find the form of a polynomial potential, which corresponds to the required type of solutions. We consider the five-dimensional gravitational model with a scalar field to demonstrate this.
\end{abstract}

\section{Introduction}

The investigations of exact special solutions of nonintegrable systems play an important role in the study of nonlinear physical phenomena. There are a few methods to construct solutions in terms of rational, hyperbolic, trigonometric or elliptic functions (all such functions are solutions of the first order polynomial differential equations) [2, 8, 9, 16, 17. 22, 23, 27, 33, 34, 35, 36, 43, 44. These methods (at least, some of them) use information about the dominant behavior of the initial system solutions in the neighbourhood of their singular points, but do not use the Laurent series representations of them. In [29] a new method has been developed as an alternative way to construct elliptic and elementary solutions and it has been shown that the use of the Laurent series solutions allows to extend the set of solutions [10] or, on the contrary, to prove non-existence of elliptic solutions [21, 42. The Laurent series solutions give the information about the global behavior of the differential system and assist in looking not only for global solutions but also for first integrals [24].

The direct algebraic method is the use of the first order polynomial differential equation, which solutions are elementary or elliptic functions, to transform the initial differential 
system in a nonlinear algebraic system in coefficients of this first order equation and parameters of the initial system. It has been proved that any finite system of algebraic equations can be solved by the Gröbner (Groebner) basis method [12, but calculations for a sufficiently complex algebraic system can be very difficult and expensive. In this paper we show that the use of the Laurent series solutions gives additional algebraic equations and allows calculations to be simplified. These equations are linear in coefficients of the first order equation and nonlinear, maybe even nonpolynomial, in parameters of the initial system. Note that one maybe should fix some of these parameters to construct the Laurent series solutions. Therefore, in contrast to the Gröbner basis method, the additional equations may be not consequences of the initial algebraic system.

To manifest the main properties of this method we consider the generalized HénonHeiles system, for which analytic and Laurent series solutions have been found in [35, 36, 39 .

\section{Painlevé analysis}

We interpret solutions of a system of ordinary differential equations (ODE's) as analytic functions, maybe with isolated singular points. A singular point of a solution is said critical (as opposed to noncritical) if the solution is multivalued (single-valued) in its neighbourhood and movable if its location depends on initial conditions [14. A singular point is said algebraic if the solution has a finite number of values in its neighbourhood. In the opposite case a singular point is either transcendental or essentially singular one.

The general solution of an ODE of order $N$ is the set of all solutions mentioned in the existence theorem of Cauchy, i.e. determined by the initial values. It depends on $N$ arbitrary independent constants. A special solution is any solution obtained from the general solution by giving values to the arbitrary constants. A system of ODE's has the Painlevé property if its general solution has no movable critical singular point 31.

The Painlevé test is any algorithm, which checks some necessary conditions for a differential equation to have the Painlevé property. The original algorithm, developed by P. Painlevé and used by him to find all the second order ODE's with the Painlevé property, is known as the $\alpha$-method. The method of S.V. Kovalevskaya [26] is not as general as the $\alpha$-method, but much more simple. In 1980, developing the Kovalevskaya method further, M.J. Ablowitz, A. Ramani and H. Segur [1] constructed a new algorithm of the Painlevé test for ODE's. This algorithm appears very useful to find solutions as a formal Laurent series [39. First of all, it allows to determine the dominant behavior of a solution in the neighbourhood of the singular point $t_{0}$. If the solution tends to infinity as $\left(t-t_{0}\right)^{\beta}$, where $\beta$ is a negative integer number, then substituting the Laurent series expansion one can transform a nonlinear differential equation into a system of linear algebraic equations on coefficients of the Laurent series. All solutions of a system depend on the parameter $t_{0}$, which characterizes the singular point location. If a single-valued solution depends on other parameters, then some coefficients of its Laurent series have to be arbitrary and the matrices in the corresponding linear systems should have zero determinants. The characteristic numbers associated with such systems (named resonances or Kovalevskaya exponents) are thus determined using the Painlevé test. In such a way we obtain solutions

only as formal series, but really we will use only a finite number of these series coefficients, 
so, we do not need the convergence of these series. At the same time for some nonintegrable systems, in particular, the generalized Hénon-Heiles system, the convergence of the Laurent- and psi-series solutions has been proved [28].

Classical results allow one to construct the suitable form of the first order autonomous polynomial equation. A theorem due to Painlevé [31, 14 proves that solutions of the equation

$$
\sum_{k=0}^{m} P_{k}(y(t), t) y_{t}(t)^{k}=0,
$$

where $y_{t}(t) \equiv \frac{\mathrm{d} y(t)}{\mathrm{d} t}, m$ is a positive integer number and $P_{k}$ are polynomials in $y(t)$ and analytic functions in $t$, have neither movable transcendental nor movable essential singular point. A theorem of Fuchs [18, 14] shows that if eq. (11) has no critical movable singular point, then the degree of $P_{k}$ as a polynomial in $y(t)$ is no more than $2 m-2 k$, in particular, $P_{m}$ does not depend on $y$.

Therefore, the necessary form of a polynomial autonomous first order ODE with the single-valued general solution is

$$
\sum_{k=0}^{m} \sum_{j=0}^{2 m-2 k} h_{j k} y^{j} y_{t}^{k}=0, \quad h_{0 m}=1,
$$

in which $m$ is a positive integer number and $h_{j k}$ are constants. A theorem of Briot and Bouquet [5, 29] proves that the general solution of (2) is either an elliptic function, or a rational function of $e^{\gamma x}, \gamma$ being some constant, or a rational function of $x$. Note that the third case is a degeneracy of the second one, which in turn is a degenerate case of the first one.

\section{The Hénon-Heiles system}

To compare methods for the construction of elliptic solutions let us consider the generalized Hénon-Heiles system with an additional non-polynomial term, which is described by the Hamiltonian:

$$
H=\frac{1}{2}\left(x_{t}^{2}+y_{t}^{2}+\lambda_{1} x^{2}+\lambda_{2} y^{2}\right)+x^{2} y-\frac{C}{3} y^{3}+\frac{\mu}{2 x^{2}}
$$

and the corresponding system of equations of motion:

$$
\left\{\begin{array}{l}
x_{t t}=-\lambda_{1} x-2 x y+\frac{\mu}{x^{3}} \\
y_{t t}=-\lambda_{2} y-x^{2}+C y^{2}
\end{array}\right.
$$

where subscripts denote derivatives: $x_{t t} \equiv \frac{\mathrm{d}^{2} x}{\mathrm{~d} t^{2}}$ and $y_{t t} \equiv \frac{\mathrm{d}^{2} y}{\mathrm{~d} t^{2}}, \lambda_{1}, \lambda_{2}, \mu$ and $C$ are arbitrary numerical parameters. Note that if $\lambda_{2} \neq 0$, then one can put $\lambda_{2}=\operatorname{sign}\left(\lambda_{2}\right)$ without the loss of generality.

If $C=1, \lambda_{1}=1, \lambda_{2}=1$ and $\mu=0$, then (4) is the original Hénon-Heiles system [19]. Emphasizing that their choice of potential does not proceed from experimental data, Hénon 
and Heiles have proposed this system to study the motion of a star in an axial-symmetric and time-independent potential, because, on the one hand, it is analytically simple and this makes the numerical computations of trajectories easy, but, on the other hand, it is sufficiently complicated to give trajectories which are far from trivial. Subsequent numerical investigations [6, 7] show that in the complex $t$-plane singular points of solutions of the Hénon-Heiles system group in self-similar spirals. It turns out that there are extremely complicated distributions of singularities, forming a boundary, across which the solutions can not be analytically continued.

By the use of Painlevé analysis the following integrable cases of the generalized HénonHeiles system have been found:

(i) $C=-1, \quad \lambda_{1}=\lambda_{2}$,

(ii) $C=-6, \quad \lambda_{1}, \lambda_{2}$ arbitrary,

(iii) $C=-16, \quad \lambda_{1}=\lambda_{2} / 16$.

In all above-mentioned cases system (4) is integrable for any value of $\mu$. The general solutions of the Hénon-Heiles system are known only in integrable cases [37, 38, 11, in other cases not only four-, but even three-parameter exact solutions have yet to be found. The generalized Hénon-Heiles system has attracted enormous attention over the years and has used as a model in astronomy [30] and in gravitation [25, 32].

\section{Methods for construction of elliptic solutions}

\subsection{The construction of the nonlinear algebraic system}

The function $y$, solution of system (44), satisfies the following fourth-order polynomial equation:

$$
\begin{aligned}
y_{t t t t} & =(2 C-8) y_{t t} y-\left(4 \lambda_{1}+\lambda_{2}\right) y_{t t}+2(C+1) y_{t}^{2}+ \\
& +\frac{20 C}{3} y^{3}+\left(4 C \lambda_{1}-6 \lambda_{2}\right) y^{2}-4 \lambda_{1} \lambda_{2} y-4 H
\end{aligned}
$$

We note that the energy of the system $H$ is not an arbitrary parameter, but a function of initial data: $y_{0}, y_{0 t}, y_{0 t t}$ and $y_{0 t t t}$. The form of this function depends on $\mu$ :

$$
H=\frac{y_{0 t}^{2}+y_{0}^{2}}{2}-\frac{C y_{0}^{3}}{3}+\frac{\lambda_{1}+2 y_{0}}{2}\left(C y_{0}^{2}-\lambda_{2} y_{0}-y_{0 t t}\right)+\frac{\left(\lambda_{2} y_{0 t}+2 C y_{0} y_{0 t}-y_{0 t t t}\right)^{2}+\mu}{2\left(C y_{0}^{2}-\lambda_{2} y_{0}-y_{0 t t}\right)} .
$$

This formula is correct only if $x_{0}=C y_{0}^{2}-\lambda_{2} y_{0}-y_{0 t t} \neq 0$. If $x_{0}=0$, which is possible only for $\mu=0$, then we can not express $x_{0 t}$ through $y_{0}, y_{0 t}, y_{0 t t}$ and $y_{0 t t t}$, so $H$ is not a function of the initial data. If $y_{0 t t t}=2 C y_{0} y_{0 t}-\lambda_{2} y_{0 t}$, then eq. (5) with an arbitrary $H$ corresponds to system (4) with $\mu=0$, in opposite case eq. (5) does not correspond to system (4).

To find a special solution of eq. (5) one can assume that $y$ satisfies some more simple equation. For example, there exist solutions in terms of the Weierstrass elliptic function (or a degenerate elliptic function), which satisfy the following first-order differential equation:

$$
y_{t}^{2}=\tilde{A} y^{3}+\tilde{B} y^{2}+\tilde{C} y+\tilde{D}
$$


where $\tilde{A}, \tilde{B}, \tilde{C}$ and $\tilde{D}$ are constants to be determined.

E.I. Timoshkova [35] generalized eq. (6) :

$$
y_{t}^{2}=A_{4} y^{3}+A_{3} y^{5 / 2}+A_{2} y^{2}+A_{1} y^{3 / 2}+A_{0} y+\tilde{D},
$$

( $A_{j}$ are constants) and found new one-parameter solutions of the Hénon-Heiles system in two nonintegrable cases $(C=-4 / 3$ or $C=-16 / 5)$. The Timoshkova's method allows one to seek both single- and multivalued solutions. For the generalized Hénon-Heiles system new solutions (i.e. solutions with $A_{3} \neq 0$ or $A_{1} \neq 0$ ) are derived only at $\tilde{D}=0$. These solutions are single-valued, because the substitution $y(t)=\varrho(t)^{2}$ gives:

$$
\varrho_{t}^{2}=\frac{1}{4}\left(A_{4} \varrho^{4}+A_{3} \varrho^{3}+A_{2} \varrho^{2}+A_{1} \varrho+A_{0}\right)
$$

Following [36, we use the substitution

$$
y(t)=\varrho(t)^{2}+P_{0},
$$

where $P_{0}$ is a constant, and transform eq. (15) into

$$
\begin{aligned}
\varrho_{t t t t} \varrho & =-4 \varrho_{t t t} \varrho_{t}-3 \varrho_{t t}^{2}+2(C-4) \varrho_{t t} \varrho^{3}+\left(2 P_{0}(C-4)-4 \lambda_{1}-\lambda_{2}\right) \varrho_{t t} \varrho+ \\
& +2(3 C-2) \varrho_{t}^{2} \varrho^{2}+\left(2 C P_{0}-4 \lambda_{1}-8 P_{0}-\lambda_{2}\right) \varrho_{t}^{2}+\frac{10}{3} C \varrho^{6}+ \\
& +\left(2 C \lambda_{1}+10 C P_{0}-3 \lambda_{2}\right) \varrho^{4}+2\left(2 \lambda_{1} C P_{0}+5 C P_{0}^{2}-\lambda_{1} \lambda_{2}-3 P_{0} \lambda_{2}\right) \varrho^{2}+ \\
& +\frac{10}{3} C P_{0}^{3}+2 \lambda_{1} C P_{0}^{2}-3 P_{0}^{2} \lambda_{2}-2 \lambda_{1} \lambda_{2} P_{0}-2 H .
\end{aligned}
$$

If $\varrho(t)$ satisfies (8), then eq. (10) is equivalent to the following system

$$
\left\{\begin{array}{l}
\left(3 A_{4}+4\right)\left(2 C-3 A_{4}\right)=0 \\
A_{3}\left(9 C-21 A_{4}-16\right)=0 \\
96 A_{4} C P_{0}-240 A_{4} A_{2}-192 A_{4} \lambda_{1}-384 A_{4} P_{0}-48 A_{4} \lambda_{2}- \\
-105 A_{3}^{2}+128 A_{2} C-192 A_{2}+128 C \lambda_{1}+640 C P_{0}-192 \lambda_{2}=0 \\
40 A_{3} C P_{0}-90 A_{4} A_{1}-65 A_{3} A_{2}-80 A_{3} \lambda_{1}- \\
-160 A_{3} P_{0}-20 A_{3} \lambda_{2}+56 C A_{1}-64 A_{1}=0 \\
16 A_{2} C P_{0}-36 A_{4} A_{0}-21 A_{3} A_{1}-8 A_{2}^{2}-32 A_{2} \lambda_{1}-64 A_{2} P_{0}-8 \lambda_{2} A_{2}+ \\
+24 C A_{0}+64 \lambda_{1} C P_{0}+160 C P_{0}^{2}-16 A_{0}-32 \lambda_{1} \lambda_{2}-96 P_{0} \lambda_{2}=0 \\
10 A_{3} A_{0}+\left(5 A_{2}+8 C P_{0}-16 \lambda_{1}-32 P_{0}-4 \lambda_{2}\right) A_{1}=0
\end{array}\right.
$$

and the equation for the energy $H$ :

$$
\begin{aligned}
H & =\frac{1}{384}\left(96 C A_{0} P_{0}-48 A_{0} A_{2}+384 C \lambda_{1} P_{0}^{2}+640 C P_{0}^{3}-9 A_{1}^{2}-\right. \\
& \left.-192 A_{0} \lambda_{1}-384 A_{0} P_{0}-48 A_{0} \lambda_{2}-384 \lambda_{1} \lambda_{2} P_{0}-576 \lambda_{2} P_{0}^{2}\right)
\end{aligned}
$$


It has been proposed [17 to seek solutions as polynomials with three arbitrary coefficients

$$
y(t)=P_{2} \varrho(t)^{2}+P_{1} \varrho(t)+P_{0},
$$

where the function $\varrho(t)$ satisfies eq. (8). The function

$$
\breve{\varrho}(t)=\frac{1}{\sqrt{P_{2}}}\left(\varrho(t)-\frac{P_{1}}{2}\right),
$$

satisfies eq. (8) as well, therefore, one and the same function $y(t)$ corresponds to a twoparameter set of coefficients $A_{i}(i=0 . .4)$ and $P_{k}(k=0 . .2)$, so we always can put

$$
P_{2}=1 \quad \text { and } \quad P_{1}=0,
$$

without loss of generality.

In the general case, when one uses Fan's technique [17] (see, for example, 45]) with polynomials of degree $L$ in solutions of eq. (8) or eq. (6), it is possible to simplify calculations, putting

$$
P_{L}=1 \quad \text { and } \quad P_{L-1}=0 .
$$

In particular, the consideration of linear combinations $P_{1} y+P_{0}$ instead of the function $y$ is useless.

System (11) has been solved by REDUCE using the standard function solve and the Gröbner basis method [36. The goal of this paper is to show that the use of the Laurent series solutions allows to obtain some solutions of (11) solving only linear systems and nonlinear equations in one variable.

We cannot use this method for arbitrary $C$, because the Laurent series solutions are different for different $C$. So, first of all we have to fix value of $C$. Note that if $A_{3}=A_{1}=0$ then $y$ satisfies eq. (6) with $\tilde{D}=0$, hence, such solutions are known. The remaining problem can be separated on two cases: $A_{3} \neq 0$ or $A_{3}=0$ and $A_{1} \neq 0$. If $A_{3} \neq 0$, then from two first equations of system (11) it follows that

$$
C=-\frac{4}{3} \quad \text { and } \quad A_{4}=-\frac{4}{3} \quad \text { or } \quad C=-\frac{16}{5} \quad \text { and } \quad A_{4}=-\frac{32}{15} .
$$

Surely, if some solution with $A_{3}=0$ corresponds to the above-mentioned values of $C$, then it can be found as well.

\subsection{Construction of linear algebraic system}

Let choose $C=-4 / 3$ (the case $C=-16 / 5$ one can consider analogously). After substitution $C=-4 / 3$ and $A_{4}=-4 / 3$ in (11) we obtain

$$
\left\{\begin{array}{l}
512 P_{0}+128 A_{2}-256 \lambda_{1}+384 \lambda_{2}+315 A_{3}^{2}=0, \\
56 A_{1}+\left(640 P_{0}+195 A_{2}+240 \lambda_{1}+60 \lambda_{2}\right) A_{3}=0, \\
63 A_{3} A_{1}+24\left(A_{2}+4 \lambda 1+\lambda_{2}+\frac{32}{3} P_{0}\right) A_{2}+ \\
+256 \lambda_{1} P_{0}+640 P_{0}^{2}+96 \lambda_{1} \lambda_{2}+288 P_{0} \lambda_{2}=0, \\
10 A_{3} A_{0}+\left(5 A_{2}-\frac{128}{3} P_{0}-16 \lambda_{1}-4 \lambda_{2}\right) A_{1}=0 .
\end{array}\right.
$$


If we consider system (17) separately from the differential equations (8) and (10), from which it has been obtained, then it would be difficult to solve system (17) without the use of the Gröbner basis method. At the same time from equations (8) and (10) we can obtain additional information, which assists us to solve system (17).

Let us construct the Laurent series solutions for eq. (10). The method of construction of the Laurent series solutions for the generalized Hénon-Heiles system has been described in detail in [39]. For eq. (10) with $C=-4 / 3$ we obtain that solutions have singularities proportional to $1 / t$ and the values of resonances are -1 (corresponding to the arbitrary parameter $\left.t_{0}\right), 1,4$ and 10. The Laurent series solutions are (we put $t_{0}=0$ ):

$$
\tilde{\varrho}= \pm\left(\frac{i \sqrt{3}}{t}+c_{0}+\frac{i \sqrt{3}}{24}\left(3 \lambda_{2}-2 \lambda_{1}+4 P_{0}+62 c_{0}^{2}\right) t+\ldots\right),
$$

where

$$
c_{0}=\frac{ \pm \sqrt{161700 \lambda_{1}-121275 \lambda_{2} \pm \sqrt{1155\left(5481 \lambda_{2}^{2}-12768 \lambda_{1} \lambda_{2}+8512 \lambda_{1}^{2}\right)}}}{2310} .
$$

Two signs " $\pm "$ in (19) are independent. At the same time, functions $\tilde{\varrho}$ and $-\tilde{\varrho}$ correspond to one and the same function $\tilde{y}$, so there are four different Laurent series solutions. The coefficients $c_{3}$ and $c_{9}$ are arbitrary. To find any number of coefficients, we should solve only two nonlinear equations in one variable and linear equations in one variable.

The algorithm of the construction of elliptic solutions from the Laurent series solutions is the following [29]:

- Choose a positive integer $m$ and define the first order ODE (2) with unknown coefficients $h_{j k}$.

- Compute coefficients of the Laurent series $\tilde{\varrho}$. The number of coefficients has to be greater than the number of unknowns.

- Substituting the obtained coefficients, transform eq. (21) into a linear and overdetermined system in $h_{j k}$ with coefficients depending on arbitrary parameters.

- Exclude $h_{j k}$ and obtain the nonlinear system in parameters.

- Solve the obtained system.

To obtain the explicit form of the elliptic function, which satisfies the known first order ODE, one can use the classical method due to Poincaré, which has been implemented in Maple as the package "algcurves" 20].

On the first step we choose eq. (2), which coincides with eq. (8). It means that $m=2$, all $h_{j 1}$ are equal to zero and all $h_{j 0}=-A_{j} / 4$. After the second and the third steps we obtain a linear system in $A_{j}$. The package of computer algebra procedures, which transforms the first order equation into a such system of algebraic equations has been written [40] in Maple.

The obtained system has the triangular form and is linear in $H, c_{3}$ and $c_{9}$ as well. From the first equation we obtain anew that $A_{4}=-4 / 3$. From the second equation it follows:

$$
A_{3}=\frac{16}{3} c_{0}
$$


and so on:

$$
\begin{aligned}
A_{2}= & -70 c_{0}^{2}-3 \lambda_{2}+2 \lambda_{1}-4 P_{0}, \\
A_{1}= & \left(\frac{40}{3} P_{0}-60 \lambda_{1}+50 \lambda_{2}+1300 c_{0}^{2}\right) c_{0}, \\
A_{0}= & -40 i \sqrt{3} c_{3}-\frac{21535}{12} c_{0}^{4}+\left(\frac{565}{6} \lambda_{1}-\frac{405}{4} \lambda_{2}-\frac{245}{3} P_{0}\right) c_{0}^{2}+ \\
& +\frac{7}{4} \lambda_{1} \lambda_{2}-\frac{21}{16} \lambda_{2}^{2}-\frac{7}{12} \lambda_{1}^{2}+\frac{7}{3} \lambda_{1} P_{0}-\frac{7}{2} \lambda_{2} P_{0}-\frac{7}{3} P_{0}^{2} .
\end{aligned}
$$

From the next equation of the system we obtain $c_{3}$ and, finally,

$$
\begin{aligned}
A_{0} & =\frac{15645}{4} c_{0}^{4}+\left(\frac{1545}{4} \lambda_{2}-465 P_{0}-\frac{1495}{2} \lambda_{1}\right) c_{0}^{2}+\frac{537}{20} \lambda_{1}^{2}- \\
& -\frac{663}{20} \lambda_{1} \lambda_{2}+\frac{729}{80} \lambda_{2}^{2}+19 \lambda_{1} P_{0}-\frac{37}{2} \lambda_{2} P_{0}-\frac{17}{3} P_{0}^{2} .
\end{aligned}
$$

Substituting the values of $A_{k}$, which correspond to one of the possible values of $c_{0}$, in system (17) we obtain that it is satisfied for all values of $\lambda_{1}, \lambda_{2}$ and $P_{0}$, so we do not need to solve the nonlinear equations. Therefore we settle the nonlinear algebraic system (17), solving only linear equations and nonlinear equation in one variable. We have used the values of only six coefficients of the Laurent series solutions. Note that, for $c_{0}$ and $-c_{0}$ we obtain one and the same values of $A_{4}, A_{2}$ and $A_{0}$, and the opposite values of $A_{3}$ and $A_{1}$. From (88) it follows that these solutions correspond to $\pm \varrho(t)$, and, hence, give one and the same function $y(t)$. Therefore not four, but two different elliptic (or degenerated elliptic) solutions of eq. (5) have been found. The elliptic solutions $y(t)$ are fourth-order elliptic functions and can be expressed in terms of the Weierstrass elliptic function $\wp\left(t-t_{0}\right)$ :

$$
y\left(t-t_{0}\right)=\left(\frac{a \wp\left(t-t_{0}\right)+b}{c \wp\left(t-t_{0}\right)+d}\right)^{2}+P_{0},
$$

where constants $a, b, c, d$ and periods of $\wp(t)$ are determined by $A_{k}$. The parameters $t_{0}$ and $P_{0}$, which defines the energy of the system, are arbitrary. Solutions of this type exist in both above-mentioned nonintegrable cases: $C=-16 / 5$ and $C=-4 / 3$. The full list of solutions is given in 36 .

\section{Multivalued solutions}

From the Painlevé theorem it follows that a solution of an autonomous polynomial first order differential equation (11) can have only two types of singular points: poles and algebraic branch points. So, the most general type of formal series solutions is the Puiseux series. Let us generalize the above method by searching for solutions, which can be expanded in Puiseux series, as follows:

$$
y=\sum_{k=-L}^{\infty} S_{k / q} t^{k / q} .
$$


We seek solutions as a polynomial

$$
y=P_{L} \rho^{L}+P_{L-1} \rho^{L-1}+P_{L-2} \rho^{L-2}+P_{L-3} \rho^{L-3}+\cdots+P_{0} .
$$

From (26) it follows that

$$
\rho=\sum_{j=-1}^{\infty} C_{j / q} t^{j / q}
$$

We assume that $\rho(t)$ satisfies the following equation:

$$
\sum_{k=0}^{m} \sum_{j=0}^{(q+1)(m-k)} h_{j k} \rho^{j} \rho_{t}^{k}=0, \quad h_{0 m}=1,
$$

where $q$ is a natural number.

To simplify calculations one can put $P_{L}=1$ and $P_{L-1}=0$ without loss of generality, because

$$
\breve{\rho}=\left(\rho-\frac{P_{n-1}}{n}\right) / \sqrt[n]{P_{n}}
$$

satisfies (29) as well. The construction of the corresponding algebraic system has been automatized in Maple [41. We plan to automatize similar construction for an arbitrary autonomous polynomial first order equation.

\section{Five-dimensional gravitational model with a scalar field}

To show how the analysis of singular behavior of solutions can assist to find the form of a potential, let us consider a five-dimensional model of a gravitational field [13, 15, with the action given by

$$
\tilde{S}=\int_{M} d^{4} x d r \sqrt{\left|\operatorname{det} \tilde{g}_{\mu \nu}\right|}\left(\frac{\tilde{R}}{4}-\frac{1}{2} \tilde{g}^{\mu \nu} \partial_{\mu} \tilde{\varphi} \partial_{\nu} \tilde{\varphi}-V(\tilde{\varphi})\right),
$$

where $M$ is the full five-dimensional space-time. The most general metric with fourdimensional Poincaré symmetry is

$$
d s^{2}=e^{2 A(r)}\left(-d x_{0}^{2}+d x_{1}^{2}+d x_{2}^{2}+d x_{3}^{2}\right)+d r^{2} .
$$

Assuming the scalar field depends only on $r$, so that $\phi=\phi(r)$, the independent equations of motion are

$$
\left\{\begin{aligned}
J^{\prime} & =-\frac{2}{3}\left(\phi^{\prime}\right)^{2}, \\
J^{2} & =\frac{1}{6}\left(\phi^{\prime}\right)^{2}-\frac{1}{3} V(\phi),
\end{aligned}\right.
$$

where $J \equiv A^{\prime} \equiv \frac{\mathrm{d} A}{\mathrm{~d} r}$ and $\phi^{\prime} \equiv \frac{\mathrm{d} \phi}{\mathrm{d} r}$. 
Similar systems of equations of motion arise also in cosmological models with a spatially flat Friedmann metric and a scalar field [4] or a phantom scalar field [3].

Let us analyse the correspondence between $\phi(r)$ and the potential $V(\phi)$. If at a singular point (fixed at the origin, without loss of generality)

$$
\phi(r) \sim \frac{1}{r^{m}}
$$

then from (33) it follows that

$$
J^{\prime} \sim \frac{1}{r^{2 m+2}} \quad \Longrightarrow \quad J \sim \frac{1}{r^{2 m+1}} \quad \Longrightarrow \quad V(\phi) \simeq V\left(\frac{1}{r^{m}}\right) \sim \frac{1}{r^{4 m+2}} .
$$

It means that solutions with simple poles can be obtained only if the degree of the polynomial potential $V(\phi)$ is equal to six. For example, let $\phi(r)=\tanh (r)$, for real $r$ this function has no singular point, but system (33) is autonomous, so if $\phi(r)$ is a solution, then $\phi\left(r-r_{0}\right)$, where $r_{0}$ is an arbitrary complex constant, is a solution as well. Then there exists $r_{0}$ such that $\tanh \left(r-r_{0}\right)$ tends to infinity as $r \rightarrow 0$, hence, the function $\tanh (r)$ cannot be a solution for any fourth degree polynomial potential. In [13] the explicit form of the sixth degree polynomial potential $V(\phi)$, which corresponds to $\tanh (r)$ has been found. Analogously one can show that if solutions tend to infinity as $1 / r^{2}$, then the degree of $V(\phi)$ is equal to 5 . If solutions tend to infinity as $1 / r^{k}$, where $k$ is a natural number greater than two, then from (34) we obtain that $V(\phi)$ cannot be a polynomial.

The polynomial potentials, which degrees are more than 6 , correspond to multivalued solutions. If solutions tend to infinity as $r^{-2 / k}$, then from (34) it is follows that the degree of $V(\phi)$ has to be equal to $4+k$. For example, if a solution in the neighbourhood of its singular points tends to infinity as $1 / \sqrt{r}$, then $k=4$ and the degree of the corresponding potential $V(\phi)$ is equal to 8 .

In conclusion of this section we say a few words about explicit solutions. Following [13] we assume that $J(r)$ is a function of $\phi$ :

$$
J(r)=-\frac{1}{3} W(\phi(r))
$$

It is straightforward to verify that system (33) is equivalent to

$$
\begin{aligned}
& \frac{\mathrm{d} \phi(r)}{\mathrm{d} r}=\frac{1}{2} \frac{\mathrm{d} W(\phi)}{\mathrm{d} \phi}, \\
& \left(\frac{\mathrm{d} W(\phi)}{\mathrm{d} \phi}\right)^{2}-\frac{1}{3} W(\phi)^{2}-V(\phi)=0 .
\end{aligned}
$$

Unfortunately the nonautonomous equation (37) can not be solved analytically. For polynomial $V(\phi)$ it may be possible to find only special solutions, e.g. $W(\phi)$ in polynomial form. For example, $W(\phi)$ cannot be a polynomial if $V(\phi)=\left(\phi^{2}-1\right)^{2}$ and we don't know a solution for this potential. At the same time, if the potential $V(\phi)=A \phi^{4}+B \phi^{2}+C$ has no double roots, then eq. (37) has a polynomial solution. 
Contrary to a scalar field theory without gravitational field, there is not a one-to-one correspondence between the form of the scalar field $\phi\left(r-r_{0}\right)$ and potential $V(\phi)$. The form of the scalar field is defined by $\frac{\mathrm{d} W}{\mathrm{~d} \phi}$, so one can add a constant to $W(\phi)$ and obtain new $V(\phi)$ for the same $\phi(r)$. On the other hand, for given $V(\phi)$ we have not one-, but two-parameter set of functions $\phi(r)$.

\section{Conclusion}

The Laurent series solutions are useful to find elliptic or elementary solutions in the analytic form. The method, proposed in 29, has been automatized and generalized on some type of multivalued solutions. It converts the local information into the global one and can be used not only as an alternative to the standard method, but also as an addition to it, which assists to find solutions of the obtained algebraic system. We have demonstrated that one can find elliptic solutions of the generalized Hénon-Heiles system solving only linear equations and nonlinear equations in one variable, instead of nonlinear system (11). At the same time, to use this method one has to know not only an algebraic system, but also the differential equations from which this system has been obtained. To find solutions of the initial ODE in the form of the Laurent or Puiseux series we use the Painleve test. This test can also assist to solve the inverse problem: to find the degree of a polynomial potential, which corresponds to the required type of solutions.

Acknowledgments. The author is grateful to I.Ya. Aref'eva, R. Conte, A.S. Koshelev, M.N. Smolyakov and I.P. Volobuev for valuable discussions. This work has been supported in part by RFBR grant 05-01-00758, Russian Federation President's grant NSh1685.2003.2 and by the Scientific Program "Universities of Russia" grant 02.02.503.

\section{References}

[1] Ablowitz M J , Ramani A and Segur H, Nonlinear evolution equations and ordinary differential equations of Painlevé type, Lett. Nuovo Cimento 23 (1978), 333-338; A connection between nonlinear evolution equations and ordinary differential equations of P-type. I \& II, J. Math. Phys. 21 (1980), 715-721, \& 1006-1015.

[2] Antonov V A and Timoshkova E I, Simple trajectories in the rotation-symmetrical gravitational field, Russ. Astron. J. 70 (1993), 265-276.

[3] Aref'eva I Ya, Koshelev A S and Vernov S Yu, Exactly Solvable SFT Inspired Phantom Model, astro-ph/0412619

[4] Barrow J D and Parsons P, New inflation from old, Class. Quantum Grav. 12 (1995), 17151721.

[5] Briot C A A and Bouquet J C, Theórie des fonctions elliptiques. Deuxiéme édition, GauthierVillars, Imprimeur-Libraire, Paris, 1875. On-line version: The Cornell Library Hist. Math. Monographs, http://historical.library.cornell.edu/

[6] Chang Y F, Tabor M, Weiss J and Corliss G, On the Analytic Structure of the Hénon-Heiles System, Phys. Lett. A $\mathbf{8 5}$ (1981), 211-213. 
[7] Chang Y F, Tabor M and Weiss J, Analytic Structure of the Hénon-Heiles Hamiltonian in integrable and nonintegrable regimes, J. Math. Phys. 23 (1982), 531-538.

[8] Conte R and Musette M, Link between solitary waves and projective Riccati equations, J. Phys. A 25 (1992), 5609-5623.

[9] Conte R and Musette M, Linearity inside nonlinearity: exact solutions to the complex Ginzburg-Landau equation, Physica D 69 (1993), 1-17.

[10] Conte R and Musette M, Solitary waves of nonlinear nonintegrable equations, chapter in the book "Dissipative solitons" (Lect. Notes Phys., Vol. 661), eds. N.N. Akhmediev and A. Ankiewicz, Springer, Berlin, 2005 arXiv:nlin.PS/0407026.

[11] Conte R, Musette M and Verhoeven C, Explicit integration of the Hénon-Heiles Hamiltonians, J. of Nonlin. Math. Phys. 12 (Supplement 1) (2005), 212-227 arXiv:nlin.SI/0412057.

[12] Davenport J H, Siret Y and Tournier E, Calcul Formel, Systemes et Algorithmes de Manipulations Algebriques, Masson, Paris, New York, 1987.

[13] DeWolfe O, Freedman D Z, Gubser S S and Karch A, Modeling the fifth dimension with scalars and gravity, Phys. Rev. D 62 (2000), 046008 arXiv:hep-th/9909134.

[14] Golubev V V, Lectures on Analytical Theory of Differential Equations, Gostekhizdat (State Pub. House), Moscow-Leningrad, 1950 \{in Russian\}.

[15] Gremm M, Four-dimensional gravity on a thick domain wall, Phys. Lett. B 478 (2000), 434438 arXiv:hep-th/9912060; Thick domain walls and singular spaces, Phys. Rev. D 62 (2000), 044017 arXiv:hep-th/0002040.

[16] Grimshaw R and Pavlov M, Exact periodic steady solutions for nonlinear wave equations: A new approach, Phys. Lett. A 251 (1999), 25-30.

[17] Fan E, An algebraic method for finding a series of exact solutions to integrable and nonintegrable nonlinear evolutions equations, J. of Phys. A 36 (2003), 7009-7026.

[18] von Fuchs L, Gesammelte mathematische Werke von L. Fuchs. Hrsg. von Richard Fuchs und Ludwig Schlesinger, Berlin, Mayer \& Müller, 1904-1909. On-line version: The Cornell Library of Historical Mathematics Monographs; http://historical.library.cornell.edu/

[19] Hénon M. and Heiles C., The applicability of the third integral of motion: some numerical experiments, Astron. J. 69 (1964), 73-79.

[20] van Hoeij M., Package 'algcurves' for Maple 6 (2000); http://www.math.fsu.edu/ hoeij/maple.html

[21] Hone A N W, Non-existence of elliptic travelling wave solutions of the complex GinzburgLandau equation, Physica D 205 (2005), 292-306.

[22] Kudryashov N A, Nonlinear differential equations with exact solutions expressed via the Weierstrass function, arXiv:nlin.CD/0312035

[23] Kudryashov N A, Simplest equation method to look for exact solutions of nonlinear differential equations, arXiv:nlin.SI/0406007

[24] Kudryashov N A, Painlevé property and the first integrals of nonlinear differential equations, arXiv:nlin.SI/0408041 
[25] Kokubun F, Gravitational waves from the Hénon-Heiles system, Phys. Rev. D 57 (1998), $2610-2612$.

[26] Kowalevski S, Sur le problème de la rotation d'un corps solide autour d'un point fixe, Acta Mathematica 12 (1889), 177-232; Sur une properiété du système d'équations différentielles qui définit la rotation d'un corps solide autour d'un point fixe, Acta Mathematica 14 (1890), 81-93, \{in French\}. Reprinted in: Kovalevskaya S V, Scientific Works, AS USSR Publ. House, Moscow, 1948, \{in Russian\}.

[27] Lenells J, Traveling Wave Solutions of the Camassa-Holm and Korteweg-de Vries Equations, J. of Nonlin. Math. Phys. 11 (2004), 508-520.

[28] Melkonian S, Psi-series solutions of the cubic Hénon-Heiles system and their convergence, $J$. Nonlin. Math. Phys. 6 (1999), 139-160 arXiv:math.DS/9904186.

[29] Musette $\mathrm{M}$ and Conte R, Analytic solitary waves of nonintegrable equations, Physica D $\mathbf{1 8 1}$ (2003) 70-79 arXiv:nlin.PS/0302051

[30] Murray C D and Dermott S F, Solar System Dynamics, University Press, Cambridge, 1999.

[31] Painlevé P., Leçons sur la théorie analytique des équations différentielles, professées à Stockholm (septembre, octobre, novembre 1895) sur l'invitation de S. M. le roi de Suède et de Norwège, Hermann, Paris, 1897; Reprinted in: Oeuvres de Paul Painlevé, V. 1, ed. du CNRS, Paris, 1973. On-line version: The Cornell Library Historical Mathematics Monographs, http://historical.library.cornell.edu/

[32] Podolský Ji and Veselý K, Chaos in pp-wave spacetime, Phys. Rev. D 58 (1998), 081501.

[33] Porubov A V and Parker D F, Some general periodic solutions to coupled nonlinear Schrödenger equations, Wave Motion 29 (1999), 97-109.

[34] Santos G S, Application of Finite Expansion in Elliptic Functions to Solve Differential Eduations, J. of the Physical Society of Japan 58 (1989), 4301-4310.

[35] Timoshkova E I, A New class of trajectories of motion in the Hénon-Heiles potential field, Astron. Zh. 76 (1999), 470-475 \{in Russian\}; Astron. Rep., 43 (1999), 406-411, \{in English\}.

[36] Timoshkova E I and Vernov S Yu, On two nonintegrable cases of the generalized Hénon-Heiles system with an additional nonpolynomial term, arXiv:math-ph/0402049

[37] Verhoeven C, Musette M and Conte R, Integration of a generalized Hénon-Heiles Hamiltonian, J. Math. Phys. 43 (2002), 1906-1915 arXiv:nlin.SI/0112030

[38] Verhoeven C, Musette M and Conte R, General solution for Hamiltonians with extended cubic and quadric potentials, Teor. Mat. Fiz. 134 (2003), 148-159 \{in Russian\}, Theor. Math. Phys. 134 (2003), 128 \{in English\} arXiv:nlin.SI/0301011.

[39] Vernov S Yu, Constructing solutions for the generalized Hénon-Heiles system through the Painlevé test, Teor. Mat. Fiz. 135 (2003), 409-419 \{in Russian\}, Theor. Math. Phys. 135 (2003), 792-801 \{in English\} arXiv:math-ph/0209063.

[40] Vernov S Yu, Construction of Single-valued Solutions for Nonintegrable Systems with the Help of the Painlevé Test, in: Proc. of the International Conference "Computer Algebra in Scientific Computing" (CASC'04, St. Petersburg, Russia, 2004), Editors: Ganzha V G, Mayr E W and Vorozhtsov E V, Technische Universitat, Munchen, Garching, 2004, 457-465 arXiv:nlin.SI/0407062. 
[41] Vernov S Yu, Construction of Exact Particular Solutions of Nonintegrable Systems by Means of Formal Laurent and Puiseux Series, Programming and Computer Software, 32 (2006), 1-7; Preprint version: Construction of global multi-valued solutions for dynamic systems, Preprint SINP MSU 2004-18/757, http://www.npi.msu.su/science.php3 \{in Russian\}.

[42] Vernov S Yu, Proof of the Absence of Elliptic Solutions of the Cubic Complex Ginzburg-Landau Equation, Theor. Math. Phys., 146 (2006), 131-139; Preprint version: On elliptic solutions of the cubic complex one-dimensional Ginzburg-Landau equation, arXiv:nlin.PS/0503009

[43] Weiss J, Bäcklund Transformation and Linearizations of the Hénon-Heiles System, Phys. Lett. A 102 (1984), 329-331.

[44] Weiss J, Bäcklund Transformation and the Hénon-Heiles System, Phys. Lett. A 105 (1984), $387-389$.

[45] Yomba E, The extended Fan's sub-equation method and its application to KdV-MkdV, BKK and variant Boussinesq equations, Phys Lett. A 336 (2005), 463-476. 